\title{
Quercetin promotes long noncoding RNA IncSHGL expression via estrogen receptor a to suppress type 2 diabetes mellitus in mice
}

\section{Hanlu Fan}

China Agricultural University

\section{Haiwen Li}

Virginia State University

Huijiao Liu

China Agricultural University

\section{Peng Li}

China Agricultural University

\section{Xiaomeng Jia}

China Agricultural University

\section{Peilan Guo}

Beijing Forestry University

\section{Qingquan Yu}

Cofoc Great Wall Chateau Sungod((huailai) Co., Ltd

\section{Xiru Li}

The General Hospital of the People's Liberation Army

\section{Yangdong Guo}

China Agricultural University

\section{Xiangdong Li ( $\nabla$ xiangdongli68@126.com)}

China Agricultural University https://orcid.org/0000-0003-2261-2393

\section{Research}

Keywords: grape pomace extract, quercertin, T2DM, ERa, IncSHGL, heterogeneous nuclear ribonucleoprotein A1 (hnRNPA1)

Posted Date: February 24th, 2020

DOl: https://doi.org/10.21203/rs.2.24284/v1

License: (c) (1) This work is licensed under a Creative Commons Attribution 4.0 International License. Read Full License 


\section{Abstract}

Background Type 2 diabetes mellitus (T2DM) is the most common type of metabolic disorder involving multiple organ systems. Grape has been reported to improve the symptoms of T2DM, the precise mechanism of its action is unclear. Our study was aimed to determine the effect and mechanism of grape pomace extract in T2DM mice induced by high fat diet (HFD). Materials and methods Ultraperformance liquid chromatography and quadrupole time-of-flight mass spectrometry were used to identify the main active compounds in grape pomace extract to improve T2DM. C57BLK/6J mice induced by HFD supplemented with or without quercetin were used to show the effects of quercetin improving T2DM. By online database research, bioinformatics analysis and molecular biology experiments, Estrogen receptor alpha (ERa)-IncSHGL (IncRNA suppressor of hepatic gluconeogenesis and lipogenesis) pathway was identify as the target for quercetin. Results Quercetin was identified as one of the most active compounds in grape pomace extract to improve T2DM. Quercetin could inhibit HFD-induced T2DM in mice by activing ERa. LncSHGL was identified as the downstream of ERa and inhibited HFD-induced T2DM. Conclusions Quercetin could be beneficial for T2DM by promoting IncSHGL transcription and activating the IncSHGL pathway, and may be used as a drug component to treat T2DM.

\section{Introduction}

Type 2 diabetes mellitus, the fifth leading cause of death in the world in 2000, is a global epidemic [1]. The prevalence of type 2 diabetes mellitus worldwide in adults old than 18 years has increased from $4.7 \%$ in 1980 to $8.5 \%$ in 2014. In 2016, type 2 diabetes mellitus was estimated to cause 1.6 million deaths [2], and it is expected to affect 366 million individuals by $2030[3,4]$. According to the reports from the Chinese Center for Disease Control and Prevention, the prevalence of type 2 diabetes mellitus in Chinese individuals older than 18 years increased from $9.7 \%$ in 2010 [5] to $10.4 \%$ in 2013 [6].

Presently, several drugs can suppress the rise of blood glucose level by maintaining it within a normal range. Because diet, activity, stress and metabolism kinetics can affect the blood glucose level, which eventually perturbing the insulin sensitivity, diabetic treatment is challenging [7]. In addition, the complexity of the treatment and the side effects of the drug itself are often unacceptable, as they negatively impact the quality of life [8]. An increasing number of individuals are turning to natural substances and foods to treat type 2 diabetes mellitus. Grapes are rich in vitamins, minerals and polyphenols, including flavonoids, proanthocyanidins, and procyanidins [9]. Many studies have shown that GPE can reduce postprandial hypertriglyceridemia and hypercholesterolemia as well as prevent insulin resistance $(\mathbb{R})[10,11]$, due to its inhibitory effects on the intestinal a-glucosidase and pancreatic a-amylase [12]. Another study showed that GSE significantly improved obesity and hyperglycemia and reduced the serum glucosamine level in obese type 2 diabetes mellitus patients [13]. In acute-dose studies, grape polyphenols were reported to reduce the serum glucose concentration in animals and humans $[14,15]$. Although studies have shown that GPE can effectively reduce hyperglycemia, the precise mechanism of action is unclear. 
With the advancement of RNA-seq technology, nearly 3 billion non-coding RNAs (nc-RNAs) accounting for $97-98 \%$ of the human genome with unclear gene functions have been discovered, $[16,17]$. Among these, $80-90 \%$ of ncRNA are longer than 200 nucleotides and not traditional protein-encoding RNAs, these ncRNAs are defined as long non-coding RNAs (IncRNAs) [18-20]. Increasing evidence has shown that IncRNAs have different biological functions, and several IncRNAs have functions in glucose and lipid metabolism [8, 21].

Here, we report that quercetin, one of the most active compounds in grape pomace extract (GPE), can improve the symptoms of HFD-induced type 2 diabetes mellitus via the regulation of IncRNA suppressor of hepatic gluconeogenesis and lipogenesis (IncSHGL). We results show that IncSHGL might be a new therapeutic target for type 2 diabetes mellitus.

\section{Materials And Methods}

\section{Mouse food preparation}

Rodent chow and western style high fat diet (HFD) were purchased from Huafukang co. (Beijing, China). For quercetin containing HFD diet, $0.1 \mathrm{~g} / \mathrm{kg}$ quercetin (Sigma, USA) of HFD, was tailor-made by the same company. All mouse food was sterilized by irradiation to minimize bacterial contamination.

Macronutrient and selected micronutrient content in the mouse HFD is summarized in Table 1.

\section{Animals}

Six-week male C57BLK/6J mice (Charles River, China) were housed in clean cages at $12 \mathrm{~h}$ light dark cycle at $20^{\circ} \mathrm{C}$ to $22^{\circ} \mathrm{C}$, with 4 mice/cage. After adaptive feeding for 1 week, all mice were randomized in each group. All animal experimental protocols are in line with the Animal Management Rules of the Ministry of Health of the People's Republic of China and the China Agricultural University Guide for the Care and Use of the Laboratory Animals. The ethical committee No. is CAU20180217-4.

\section{Type 2 diabetes mellitus mouse model}

Type 2 diabetes mellitus mouse model was reported by Houssay and colleagues previously [22, 23]. Briefly, the islet B cells were kept in a high-load state for a long time through a HFD, and finally induced a mouse model characterized by obesity, impaired glucose tolerance, IR, and abnormal lipid metabolism [22]. The model has been reported to mimic the early human and progression of type 2 diabetes mellitus [24].

\section{Purification and identification of active compounds by ultra-performance liquid chromatography (UPLC) combined with a quadrupole time-of-flight mass spectrometry (Q-TOF-MS)}

UPLC system and chromatographic conditions and identification of chemical constituents by UPC2/QTOF-MSE analysis the Waters ACQUITY UPLC system (Acquity UltraPerformance Liquid Chromatography ${ }^{\mathrm{TM}}$, USA). Grape pomace was analyzed for its chemical profiles using a Waters Xevo G2 
Q-TOF mass spectrometer (Milford, USA). To collect data in TOF MS experiments, MSE technology was used in which two separate scan functions were programmed for the MS acquisition method. Data were acquired and analyzed with Waters MassLynx v4.1 software. The sample analysis was performed in triplicate to test the repeatability.

\section{Experimental group assignment}

In this experiment, since body weight is a critical experimental indicator, a block randomization method was used. Briefly, the mice were divided into 4 zones according to their weight from light to heavy, and then grouped according to the random number table.

In the pre-experiment, the effective dose for GPE in type 2 diabetes mellitus mouse model was optimized at $2.4 \mathrm{~g} / \mathrm{Kg}$. As the median lethal dose of quercetin for mice was about $160 \mathrm{mg} / \mathrm{kg}$ [25], and a single intravenous injection of $100-150 \mathrm{mg} / \mathrm{kg}$ of quercetin was reported did not have any symptoms [26] 100 $\mathrm{mg} / \mathrm{kg}$ quercetin in HFD was chosen in this study. Each mouse was randomly assigned to one of the following groups: 1) group-1, mice fed with regular normal chow (NC) and served as control $(n=16) ; 2)$ group-2, mice fed with HFD ( $\mathrm{n}=16)$; 3) group-3, mice fed with HFD+2.4 g/kg GPE (GHFD, n=16); 4) group4 , mice fed with HFD+100 mg/kg quercetin (QHFD, $\mathrm{n}=16$ ). All mice were fed with the respective diets for 12 weeks with water available ad libitum.

\section{Food intake, body weight and fasting glucose measurements during the experiment}

During the 12-week feeding period, food intake was recorded daily, and body weight was recorded weekly. Fasting blood glucose levels were also monitored weekly using a blood glucometer and the accompanying test strips (ACCU-CHEK Meter®, Germany).

\section{Mouse glucose tolerance test (GTT)}

Briefly , the mice were fasted overnight, about $12-15 \mathrm{~h}$; glucose solution (20\%) was prepared, syringe, blood glucose meter, blood glucose test strip, timer and other laboratory supplies were prepared; The body weight of each mouse was recorded and fasting blood glucose was measured at $0 \mathrm{~min}$. The volume of intraperitoneal injection of $20 \%$ glucose solution per mouse by $2 \mathrm{~g}$ glucose per kg body weight was calculated, as follows: $20 \%$ glucose $(\mu \mathrm{l})=10 \times$ body weight $(\mathrm{g})$. After the glucose was injected, the blood glucose was measured at $15,30,60,90,120$ min by cutting off the tail of each mouse and recorded, respectively.

\section{Mouse insulin tolerance test (ITT)}

Briefly, the mice were fasted for $5 \mathrm{~h}$; the short-acting insulin stock solution (100 U/ml, Eli Lilly) was prepared, syringes, blood glucose meters, blood glucose test strips and other laboratory supplies were got ready. The body weight of each mouse was recorded and fasting blood glucose was measured at 0 min. The volume of insulin injected intraperitoneally in each mouse by injecting $0.75 \mathrm{U}$ per $\mathrm{kg}$ body weight was 
calculated. After injected with insulin, the mice were measured blood glucose at 15, 30, 60, 90, 120 min by cutting off the tail of each mouse and recorded, respectively.

\section{Animal sacrifice and tissue collection}

The mice were then injected intraperitoneally with $40 \mathrm{mg} / \mathrm{kg}$ Ketamine. Blood samples were collected and the serum was separated. Liver, heart, kidney, epididymal fat and pancreas were dissected, weighed and immediately frozen in liquid nitrogen and stored at $-80^{\circ} \mathrm{C}$, respectively.

\section{Histological analysis of animal adipocytes}

Freshly isolated epididymal fat and liver were fixed in $10 \%$ formalin (Sigma, USA) for $24 \mathrm{~h}$ followed by embedding the tissues in paraffin (Citoglas, China). The paraffin-embedded samples were sectioned and stained with hematoxylin-eosin following the procedures used by Ma et al., 2016 [27].

\section{Mouse primary hepatocyte isolation}

Hepatocytes were isolated from adult male C57BLK/6J mice, using a two-step collagenase perfusion procedure reported previously .

\section{Cell culture}

The human hepatoma cell line $\mathrm{HepG}_{2}$, human renal epithelial cell line 293T and the mice normal liver cell line NCTC-149 were obtained from the Cell Resource Center, Peking Union Medical College (licensed from ATCC). The HepG 2 cell line and 293T cell line were maintained in Dulbecco's modified Eagle's medium high glucose (DMEM-HG, Gibco Invitrogen, USA) supplemented with $10 \%$ fetal bovine serum (FBS, Gibco Invitrogen, USA). The NCTC-149 cell line was maintained in DMEM-HG supplemented with $10 \%$ horse serum. The mouse primary hepatocyte was maintained in Roswell Park Memorial Institute - 1640 (RPMI1640, Gibco Invitrogen, USA) supplemented with 10\% FBS. 100 units/ml penicillin (Sigma, USA), 100 $\mu \mathrm{g} / \mathrm{ml}$ streptomycin (Sigma, USA), and $0.16 \% \mathrm{NaHCO}_{3}$ (Sigma, USA) was added to all cell culture media. All cells were incubated in a humidified atmosphere of $95 \% \mathrm{O}_{2}$ and $5 \% \mathrm{CO}_{2}$ at $37{ }^{\circ} \mathrm{C}$.

\section{Cell processing}

When the cells were grown to about $70 \%$, the cells were treated with quercetin, palmitic acid (P), estradiol $\left(E_{2}\right), I C l 182780(\mathrm{ICl})$, propylpyrazoletriol (PPT), and diarylpropionitrile (DPN), and samples were taken 24 $\mathrm{h}$ later for subsequent experiments. All of the reagents used above were purchased from Sigma (Sigma, USA). The working concentration of quercetin is $10 \mu \mathrm{M}$. The working concentration of $\mathrm{P}$ is $500 \mu \mathrm{M}$. The working concentrations of $\mathrm{E}_{2,}, \mathrm{ICl}$, PPT and DPN are both $10^{-9} \mathrm{M}$.

\section{Construction of plasmids}


The coding sequence (CDS) of mice ERa and ER $\beta$ were obtained from CDNA of mice by PCR. ERa was inserted into pCDNA3.0 through endonuclease site ECoRI and BamHI. ER $\beta$ was inserted into pCDNA3.0 through endonuclease site EcoRI and Xhol. The promoter of mouse IncSHGL was obtained from the mouse genomic DNA by PCR and inserted into pGL3 vector by endonuclease site HindIII and Kpnl. All of the endonucleases used above were purchased from NEB Corporation of the USA.

\section{Transfection and luciferase activity analysis}

Approximately $\varangle 3 \times 10^{5}$ cells were seeded per well in a 6-well plate. When the cells were grown to about $70 \%$, plasmids were transfected into 293T cell line using PEI (Polyscience, USA) transfection reagent according to the manufacturer's protocol. pGL3- LncSHGL-promoter vector and ERa (with or without) or ERß (with or without) overexpression vector were transfected into 293T cell line, and pGL3 basic vector was used as a negative control. Luciferase activity was detected by using Firefly Luciferase Detection Kit (YPH-bio, China) through Microplate reader (Bio-tec, USA) $48 \mathrm{~h}$ later.

\section{RNA extraction and reverse transcription-quantitative PCR (RT-qPCR)}

Total RNA was extracted from cells and tissues using TRIzon reagent (Kangwei Biomedical Technology, China). The total RNA was reversed transcribed by M-MLVRT (Takara Biomedical Technology, Japan). Relative expression was calculated by using $\triangle \triangle C t(A B I, U S A)$. Sequences of the primers used for this reaction are in Table 2.

\section{Protein extraction and western blotting}

Whole cells were lysed in RIPA buffer with $1 \mathrm{mM}$ PMSF (Sigma, USA), $0.5 \%$ sodium deoxy cholate. Extracted proteins were resolved by $12 \%$ SDS-PAGE and transferred to a poiyvinylidenedifluoride (PVDF, Pall, USA) membrane. Then proteins were blocked with TBS-T containing $5 \%$ skim milk (Biotopped, China) at RT for $1 \mathrm{~h}$ and incubated overnight at $4^{\circ} \mathrm{C}$ with the primary antibody (1:1000). The PVDF membrane was washed in TBS-T and incubated for $2 \mathrm{~h}$ at room temperature with secondary antibody (1:5000). The bands were detected using enhanced chemiluminescence analysis system (GE, USA), and quantified by densitometry with Amersham Imager 600 uv (GE, USA) software. For quantitation of phosphorylated protein, phosphorylated protein was normalized to the corresponding total protein and then was normalized to the control value.

\section{Antibodies}

The specific primary antibodies were purchased from Cell Signaling Technology (CST, USA) and Snata Cruz (Snata Cruz, USA). The secondary antibodies were purchased from Beijing zhongshanJinqiao Biotechnology Co., Ltd, China. The antibodies were listed in the Table 3.

\section{Online analysis}


The main mode of action (MOA) of quercetin as an estrogen receptor agonist was analysed from the "Large Scale Visualization of Drug Induced Transcriptomic Signatures"

(http://amp.pharm.mssm.edu/dmoa/report/BRD-K97399794) website. The LncSHGL promoter sequence was searched from the website of the National Center for Biotechnology Information (NCBI, https://www.ncbi.nlm.nih.gov/). Binding sites for LncSHGL and ER a were predicted on the Jasper website (http://jaspar.genereg.net/analysis).

\section{Correlation analysis}

The Fragments Per Kilobase of exon model per Million mapped fragments (FPKM) expression data of liver cancer was download from TCGA database. The data was transformed from FPKM to TranscriptsPerKilobase of exonmodel per Million mapped reads (TPM). Genes or ncRNA related to diabetes were obtained by KEGG pathway analysis and literature search. Expression correlations between ESR1 and these genes or ncRNA were calculated and plotted in R package ggplot2.

\section{Statistical analysis}

The data were analyzed for statistical significance with the SPSS 12.0.1 Package (SPSS Inc., USA). Briefly, data for all groups were first tested for normality with the SPSS software. Before analyzing each set of data, the data was tested for their normal distribution by SPSS software, and all data were in a normal distribution. Homogeneity of variance test was used to test the homogeneity of the variance of the data before performing the analysis of variance. In the homogeneity test of variance, the variance of each group was equal. A one-way analysis of variance was tested. $P$ values $<0.05$ were regarded as statistically significant. All values are presented as the means \pm SEM (standard error of mean).

\section{Results}

\section{Quercetin, one of the most abundant active compounds in GPE, inhibits HFD-induced type 2 diabetes mellitus in mice}

GPE was found to inhibit HFD-induced type 2 diabetes mellitus (Fig. 1). GPE significantly reduced HFDinduced weight gain and fasting serum glucose level in mice (Fig. 1A, B). Additionally, GPE significantly reduced the weight of epididymal fat in HFD-induced mice (Fig. 1E). We also examined the food intake of mice and the expression changes of food intake-related genes in the hypothalamus of mice. As show in Fig. 1C and Fig. 1D, GPE did not change the intake of food. To further understand the anti-diabetic effects, GPE was analyzed by ultra-performance liquid chromatography (UPLC) and quadrupole time-of-flight mass spectrometry (Q-TOF-MS) (Fig. 1F). We tested the inhibitory efficiency of several major components in grape extract on a-galactosidase, and found that among these components, quercetin can significantly inhibit a-galactosidase activity (Fig. 1G).

To examine the major efficacy of quercetin, $0.1 \mathrm{~g} / \mathrm{kg}$ quercetin was added to the HFD. No change in the body weight was observed in mice fed NC/quercetin. A daily intake of HFD was 1.54-2.1g, the energy per 
gram of HFD was $4.057 \mathrm{Kcal}$; therefore each mouse consumed 6.25-8.52 Kcal per day. No difference in the intake of food between quercetin-treated group and control was noticed (Fig. 2C). However, quercetin improved hyperglycemia (Fig. 2A, B), IGT and IR in HFD-induced mice (Fig. 2D, E). Similar to GPE, quercetin significantly reduced the liver weight and the gonadal lipid content in HFD-induced mice (Fig. 2F). Quercetin significantly inhibited the HFD-induced body fat increase, as well as significantly inhibited the HFD-induced muscle percentage decrease (Fig. 2G). Quercetin can significantly reduce hepatic steatosis and gonadal coronal structures in HFD-induced mice (Fig. 2H). Moreover, by immunocytochemistry (IHC) analysis of the UCP1 (a marker for brown fat), we observed that quercetin can improve the whitening of brown fat in mice induced by HFD (Fig. 3A).

\section{Quercetin significantly increases the expression of diabetes-associated IncRNA through ER signal}

By using the "Large Scale Visualization of Drug Induced Transcriptomic Signatures" website, we noticed that quercetin is an estrogen receptor agonist; this was the verified data from the cell experiments (Fig. 3B).

Recently, a group of transcriptome sequencing analyses showed that LncRNA transcripts were found in the intergenic regions of the estrogen receptors binding site, and play a fundamental role in estrogendependent transcriptional responses [28], and are prerequisite conditions for the regulation of the downstream network of the steroid receptors [29, 30]. ERs have been reported to inhibit diabetes, but the molecular mechanism is unclear [31]. Through literature search and KEGG pathway analysis, genes and ncRNAs related to diabetes were obtained and calculated the correlation with ERa in TCGA liver cancer dataset (supplementary table 1 and supplementary Fig. 1). The correlation between ER $a$ and these genes was plotted in table S1 and Fig. S1. SLC2A2, B4GALT1-AS1, FAS, IRS1, HNF4A and PIK3R1 were found to be significantly correlated with ER a $(R>0.2, P<0.05)$. We examined the expression changes of these genes on the human liver cancer cell line HepG2 and found that quercetin can significantly increase the expression of B4GAT1-AS1 (Fig.S2). Next, we verified on mice liver and found that among these related genes; quercetin can significantly increase LncSHGL expression (Fig. S3). Several IncRNAs are involved in the etiology of type 2 diabetes mellitus and its complications [17, 32-34]. To investigate whether quercetin elicits its anti-diabetic effects via IncRNAs, we measured the levels of IncRNAs in HFD-induced and quercetin-treated mice. The results showed an increased IncSHGL expression after quercetin treatment (Fig. 3C). A previous study has reported that IncSHGL induces AKT phosphorylation by recruiting heterogeneous nuclear ribonucleoprotein A1 (hnRNPA1), thereby improving type 2 diabetes mellitus symptoms in mice [17]. Quercetin increased hnRNPA1 expression in the QHFD group compared with HFD gruop (Fig. 3D). Moreover, we observed that quercetin can increase the expression of IncSHGL and hnRNPA1 in the livers of mice (Fig. 3C, D).

Through the Jasper website, we noticed that ERa has the predicted binding sites within the IncSHGL promoter region (Fig. 3E). To examine the quercetin-mediated mechanism responsible for the improvement of type 2 diabetes mellitus symptoms in mice fed a HFD, we carried out several experiments. We found that quercetin increased the expression of IncSHGL and hnRNPA1 in mouse 
primary hepatocytes, mouse normal liver cell line NCTC-149 cells, and human hepatoma HepG2 cells. Furthermore, quercetin and the ER agonist increased the expression of IncSHGL and hnRNPA1 in the palmitic acid treated cells (Fig. 4A-4F). With regard to quercetin, opposing effects were observed in the presence of ER antagonists (Fig. 4A-4F). Palmitic acid decreased the phosphorylated AKT level in HepG2 cells, whereas quercetin increased it (Fig. 4G). Taken collectively, the results show that quercetin can activate AKT signaling by promoting the expression of IncSHGL and hnRNLA1, thereby inhibiting HFDinduced type 2 diabetes mellitus.

\section{Quercetin increases IncSHGL expression by ERa}

Data analysis performed with online tools indicated that quercetin is an ER agonist. As shown in Fig. 3B, both $E_{2}$ and $E R$ agonists significantly increased IncSHGL expression in cells, whereas ER antagonists decreased IncSHGL expression. We predicted the possible binding regions of ER $a$ and IncSHGL (Fig. 3E). We constructed the predicted IncSHGL promoter-binding region into a luciferase expression vector, cotransfected cells with ERa and ERß overexpression vectors, and measured the fluorescence. The results of the double luciferase experiment showed that quercetin and estrogen can increase the binding of ER a and LncSHGL promoter regions (Fig. 5A). The fluorescent signal was significantly higher in cells transfected with the ER a overexpression vector than in those transfected with the ER $\beta$ overexpressing vector (Fig. 5B). We mutated the predicted binding site in the promoter region of IncSHGL, and then cotransfected cells with ERa to detect the luciferase activity. As shown in Fig. 5C, luciferase expression was significantly reduced following mutation of the binding site of the promoter region of IncSHGL.

\section{Discussion}

With increasing daily responsibilities and challenges, many individuals do not pay adequate attention to their health. For instance, many individuals are obese, which can cause type 2 diabetes mellitus, due to poor dietary choices and unreasonable social pressures. Currently, type 2 diabetes mellitus is a major disease that can severely endanger human health. Several drugs can effectively suppress the increased serum glucose levels in patients. However, the side effects of these drugs cannot be ignored, as they are a concern for many individuals. Several studies have reported that natural compounds, such as those found in grapes, can curb the symptoms of type 2 diabetes mellitus and other metabolic syndromes, but their mechanisms of action are unclear [35-37]. Consistent with the results of other studies, we report that GPE suppressed the symptoms of HFD-induced type 2 diabetes mellitus. However, the mechanism of action remains unclear.

By UPLC and Q-TOF-MS, we identified quercetin to be one of the most active compounds in GPE. Quercetin is a flavonoid. These compounds have received much attention due to their structural diversity, richness, strong pharmacological activity, and safety [38]. Numerous studies have reported the biological effects of quercetin, and its potential benefits as an anti-cancer, anti-oxidative, and anti-inflammatory agent, especially in the treatment of type 2 diabetes mellitus [39-42]. Quercetin can improve the symptoms and eliminate the complications of diabetes [38, 43,44]. Liu and colleagues have reported the 
involvement of quercetin in the insulin-mediated regulation of the glucose level in skeletal muscle through AMPK [43]. Soo-Mi and colleagues have demonstrated that quercetin can improve hyperglycemia and dyslipidemia in type 2 diabetes mellitus $\mathrm{db} / \mathrm{db}$ mice [44]. Consistent with the results of previous studies, our findings indicated that quercetin reduced HFD-induced hyperglycemia and improved IR.

Recently, a group of transcriptome sequencing analyses showed that LncRNA transcripts were found in the intergenic regions of the estrogen receptors binding site, and play a fundamental role in estrogendependent transcriptional responses [28], and are prerequisite conditions for the regulation of the downstream network of the steroid receptors $[29,30]$. Several IncRNAs are involved in the etiology of type 2 diabetes mellitus and its complications [17, 32-34]. To define the mechanism of action by which quercetin improved the symptoms of type 2 diabetes mellitus in mice fed a HFD, we followed previous studies that have reported an association between IncRNAs and type 2 diabetes mellitus [45-47]. LncRNA have key roles in various biological processes $[16,19]$. For example, seven IncRNAs have been described to function in metabolic processes in the liver. We report that quercetin significantly increased IncSHGL expression after validating the expression of the seven IncRNAs in the livers of the four groups of mice. LncSHGL sequesters hnRNPA1 to inhibit hepatic gluconeogenesis and lipogenesis, which improves the symptoms of diabetes [17].

Our in vitro and in vivo results demonstrate that quercetin improved the symptoms of type 2 diabetes mellitus in mice fed a HFD. In addition, our results illustrate for the first time an association between ERa and IncSHGL. Quercetin increased IncSHGL expression by promoting the binding of ERa to the IncSHGL promoter region.

The limitation of this study is that it lacks clinical data; essentially, there is no evidence to indicate that quercetin is effective in diabetic patients. The effects of quercetin in diabetic patients will be investigated in a subsequent study.

Here, we reported the mechanism of action by which GPE improved the symptoms of type 2 diabetes mellitus in mice fed a HFD. Quercetin is one of the most active compounds in GPE, and it promoted ERa binding to the IncSHGL promoter region, thereby increasing IncSHGL expression and suppressing the symptoms of type 2 diabetes mellitus induced by a HFD (Fig. 5C). Our results show that quercetin could be beneficial for type 2 diabetes mellitus by promoting IncSHGL transcription and activating the IncSHGL pathway, and may be used as a drug component to treat type 2 diabetes mellitus.

\section{Conclusions}

We found that GPE could improve T2DM and identified quercetin as one of the most abundant and active compounds in GPE. Quercetin could inhibit HFD-induced T2DM in mice by activing ERa. LncSHGL was identified as the downstream of ERa and inhibited HFD-induced T2DM. Our results show that quercetin could be beneficial for T2DM by promoting IncSHGL transcription and activating the IncSHGL pathway, and may be used as a drug component to treat T2DM. 


\section{Abbreviations}

DPN

diarylpropionitrile

$\mathrm{ERa}$

estrogen receptora

ERB

estrogen receptor $\beta$

ERs

estrogen receptors

E2

estradiol

FPKM

Fragments Per Kilobase of exon model per Million mapped fragments

GSE

Grape seed extract

GPE

Grape pomace aqueous

HFD

high fat diet

hnRNPA1

heterogeneous nuclear ribonucleoprotein A1

$\mathrm{ICl}$

ICI 182780

IHC

immunocytochemistry

IR

insulin resistance

LncRNA

long non-coding RNA

LnCSHGL

IncRNA suppressor of hepatic gluconeogenesis and lipogenesis

PPT

propylpyrazoletriol

Q-TOF-MS

quadrupole time-of-flight mass spectrometry

TPM

Transcripts Per Kilobase of exonmodel per Million mapped reads

T2DM

type 2 diabetes mellitus 
UPLC

ultra-performance liquid chromatograph

\section{Declarations}

\section{Ethics approval and consent to participate}

All animal experimental protocols are in line with the Animal Management Rules of the Ministry of Health of the People's Republic of China and the China Agricultural University Guide for the Care and Use of the Laboratory Animals. The ethical committee No. is CAU20180217-4.

\section{Consent for publication}

Not applicable.

\section{Availability of data and materials}

The datasets used and/or analysed during the current study are available from the corresponding author on reasonable request.

\section{Competing interests}

The authors declare that they have no competing interests.

\section{Funding}

No funding.

\section{Author's Contributions}

Fan hanlu and Li xiangdong designed these experiments; Fan hanlu, Li haiwen, Liu huijiao, Li peng, Jia xiaomeng and Guo peilan did these experiments; Fan hanlu and Li xiangdong wrote the main manuscript text; Fan hanlu prepared figures. All authors reviewed the manuscript.

\section{Acknowledgements}

This work was supported by State Key Laboratory of Agro biotechnology grants (2018SKLAB6-19).

\section{References}

1. Roglic, G., et al., The burden of mortality attributable to diabetes: realistic estimates for the year 2000. Diabetes Care, 2005. 28(9): p. 2130-5.

2. WHO, 2018. 
3. Rathmann, W. and G. Giani, Global prevalence of diabetes: estimates for the year 2000 and projections for 2030. Diabetes Care, 2004. 27(10): p. 2568-9; author reply 2569.

4. Wild, S., et al., Global prevalence of diabetes: estimates for the year 2000 and projections for 2030. Diabetes Care, 2004. 27(5): p. 1047-53.

5. Xu, Y., et al., Prevalence and control of diabetes in Chinese adults. JAMA, 2013. 310(9): p. 948-59.

6. Wang, L., et al., Prevalence and Ethnic Pattern of Diabetes and Prediabetes in China in 2013. JAMA, 2017. 317(24): p. 2515-2523.

7. Varanko, A.K. and A. Chilkoti, Molecular and Materials Engineering for Delivery of Peptide Drugs to Treat Type 2 Diabetes. Adv Healthc Mater, 2019: p. e1801509.

8. Eton, D.T., et al., A systematic review of patient-reported measures of burden of treatment in three chronic diseases. Patient Relat Outcome Meas, 2013. 4: p. 7-20.

9. Weber, H.A., et al., Comparison of proanthocyanidins in commercial antioxidants: grape seed and pine bark extracts. J Agric Food Chem, 2007. 55(1): p. 148-56.

10. Meeprom, A., et al., Grape seed extract supplementation prevents high-fructose diet-induced insulin resistance in rats by improving insulin and adiponectin signalling pathways. Br J Nutr, 2011. 106(8): p. 1173-81.

11. Suwannaphet, W., et al., Preventive effect of grape seed extract against high-fructose diet-induced insulin resistance and oxidative stress in rats. Food Chem Toxicol, 2010. 48(7): p. 1853-7.

12. Adisakwattana, S., et al., Evaluation of alpha-glucosidase, alpha-amylase and protein glycation inhibitory activities of edible plants. Int J Food Sci Nutr, 2010. 61(3): p. 295-305.

13. Kar, P., et al., Effects of grape seed extract in Type 2 diabetic subjects at high cardiovascular risk: a double blind randomized placebo controlled trial examining metabolic markers, vascular tone, inflammation, oxidative stress and insulin sensitivity. Diabet Med, 2009. 26(5): p. 526-31.

14. Torronen, R., et al., Berries modify the postprandial plasma glucose response to sucrose in healthy subjects. Br J Nutr, 2010. 103(8): p. 1094-7.

15. Sapwarobol, S., et al., Postprandial blood glucose response to grape seed extract in healthy participants: A pilot study. Pharmacogn Mag, 2012. 8(31): p. 192-6.

16. Consortium, E.P., An integrated encyclopedia of DNA elements in the human genome. Nature, 2012. 489(7414): p. 57-74.

17. Wang, J., et al., Long Noncoding RNA IncSHGL Recruits hnRNPA1 to Suppress Hepatic Gluconeogenesis and Lipogenesis. Diabetes, 2018. 67(4): p. 581-593.

18. Bertone, P., et al., Global identification of human transcribed sequences with genome tiling arrays. Science, 2004. 306(5705): p. 2242-6.

19. Kapranov, P., et al., RNA maps reveal new RNA classes and a possible function for pervasive transcription. Science, 2007. 316(5830): p. 1484-8.

20. Huarte, M., The emerging role of IncRNAs in cancer. Nat Med, 2015. 21(11): p. 1253-61. 
21. Goyal, N., et al., Long non-coding RNA H19 inhibition promotes hyperglycemia in mice by upregulating hepatic FoxO1 levels and promoting gluconeogenesis. J Mol Med (Berl), 2019. 97(1): p. 115-126.

22. Houssay, B.A. and C. Martinez, Experimental Diabetes and Diet. Science, 1947. 105(2734): p. 548-9.

23. Wang, C., et al., FAM3A activates PI3K p110alpha/Akt signaling to ameliorate hepatic gluconeogenesis and lipogenesis. Hepatology, 2014. 59(5): p. 1779-90.

24. Winzell, M.S. and B. Ahren, The high-fat diet-fed mouse: a model for studying mechanisms and treatment of impaired glucose tolerance and type 2 diabetes. Diabetes, 2004. 53 Suppl 3: p. S215-9.

25. Sullivan, M., R.H. Follis, Jr., and M. Hilgartner, Toxicology of podophyllin. Proc Soc Exp Biol Med, 1951. 77(2): p. 269-72.

26. Ambrose, A.M., D.J. Robbins, and F. Deeds, Comparative toxicites of quercetin and quercitrin. J Am Pharm Assoc Am Pharm Assoc, 1952. 41(3): p. 119-22.

27. Ma, W., et al., Zika Virus Causes Testis Damage and Leads to Male Infertility in Mice. Cell, 2016. 167(6): p. 1511-1524 e10.

28. Hah, N., et al., A rapid, extensive, and transient transcriptional response to estrogen signaling in breast cancer cells. Cell, 2011. 145(4): p. 622-34.

29. Yang, L., et al., IncRNA-dependent mechanisms of androgen-receptor-regulated gene activation programs. Nature, 2013. 500(7464): p. 598-602.

30. Knoll, M., H.F. Lodish, and L. Sun, Long non-coding RNAs as regulators of the endocrine system. Nat Rev Endocrinol, 2015. 11(3): p. 151-60.

31. Tiano, J.P., et al., Estrogen receptor activation reduces lipid synthesis in pancreatic islets and prevents beta cell failure in rodent models of type 2 diabetes. J Clin Invest, 2011. 121(8): p. 3331-42.

32. Shang, J., et al., Identification of key IncRNAs contributing to diabetic nephropathy by gene coexpression network analysis. Sci Rep, 2019. 9(1): p. 3328.

33. Thomas, A.A., et al., IncRNA H19 prevents endothelial-mesenchymal transition in diabetic retinopathy. Diabetologia, 2019. 62(3): p. 517-530.

34. Yu, J.L., et al., Downregulation of long noncoding RNA H19 rescues hippocampal neurons from apoptosis and oxidative stress by inhibiting IGF2 methylation in mice with streptozotocin-induced diabetes mellitus. J Cell Physiol, 2018.

35. Costabile, G., et al., Grape pomace polyphenols improve insulin response to a standard meal in healthy individuals: A pilot study. Clin Nutr, 2018.

36. Van Hul, M., et al., Reduced obesity, diabetes, and steatosis upon cinnamon and grape pomace are associated with changes in gut microbiota and markers of gut barrier. Am J Physiol Endocrinol Metab, 2018. 314(4): p. E334-E352.

37. Griffin, L.E., et al., Alterations to metabolically active bacteria in the mucosa of the small intestine predict anti-obesity and anti-diabetic activities of grape seed extract in mice. Food Funct, 2017. 8(10): p. 3510-3522. 
38. Shi, G.J., et al., In vitro and in vivo evidence that quercetin protects against diabetes and its complications: A systematic review of the literature. Biomed Pharmacother, 2019. 109: p. 1085-1099.

39. Zhuang, M., et al., Islet protection and amelioration of type 2 diabetes mellitus by treatment with quercetin from the flowers of Edgeworthia gardneri. Drug Des Devel Ther, 2018. 12: p. 955-966.

40. Ma, Y.Y., et al., alpha-Glucosidase Inhibition and Antihyperglycemic Activity of Phenolics from the Flowers of Edgeworthia gardneri. J Agric Food Chem, 2015. 63(37): p. 8162-9.

41. Lamson, D.W. and M.S. Brignall, Antioxidants and cancer, part 3: quercetin. Altern Med Rev, 2000. 5(3): p. 196-208.

42. Vessal, M., M. Hemmati, and M. Vasei, Antidiabetic effects of quercetin in streptozocin-induced diabetic rats. Comp Biochem Physiol C Toxicol Pharmacol, 2003. 135C(3): p. 357-64.

43. Liu, K., et al., Quercetin oppositely regulates insulin-mediated glucose disposal in skeletal muscle under normal and inflammatory conditions: The dual roles of AMPK activation. Mol Nutr Food Res, 2016. 60(3): p. 551-65.

44. Jeong, S.M., et al., Quercetin ameliorates hyperglycemia and dyslipidemia and improves antioxidant status in type 2 diabetic $d b / d b$ mice. Nutr Res Pract, 2012. 6(3): p. 201-7.

45. Zhang, X., et al., Microarray analysis reveals long noncoding RNA SOX2OT as a novel candidate regulator in diabetic nephropathy. Mol Med Rep, 2018. 18(6): p. 5058-5068.

46. Li, S.Y. and K. Susztak, The long noncoding RNA Tug1 connects metabolic changes with kidney disease in podocytes. J Clin Invest, 2016. 126(11): p. 4072-4075.

47. Long, J., et al., Long noncoding RNA Tug1 regulates mitochondrial bioenergetics in diabetic nephropathy. J Clin Invest, 2016. 126(11): p. 4205-4218.

\section{Tables}


Table 1. Macronutrient and selected micronutrient content in the mouse high fat diet

\begin{tabular}{|c|c|c|}
\hline & $\mathrm{gm} \%$ & $\mathrm{kcal} \%$ \\
\hline Protein & 26.2 & 20 \\
\hline Carbohydrate & 26.3 & 20 \\
\hline Fat & 34.9 & 60 \\
\hline Ingredient & $\mathrm{gm}$ & kcal \\
\hline Casein, 30 Mesh & 200 & 800 \\
\hline L-Cystine & 3 & 12 \\
\hline Corn Starch & 0 & 0 \\
\hline Maltodextrin 10 & 125 & 500 \\
\hline Sucrose & 68.8 & 275.2 \\
\hline Cellulose, BW200 & 50 & 0 \\
\hline Soybean Oil & 25 & 225 \\
\hline Lard* & 245 & 2205 \\
\hline Mineral Mix S10026 & 10 & 0 \\
\hline DiCalcium Phosphare & 13 & 0 \\
\hline Calcium Carbonate & 5.5 & 0 \\
\hline Potassium Cirate, $1 \mathrm{H}_{2} \mathrm{O}$ & 16.5 & 0 \\
\hline Vitamin Mix V10001 & 10 & 40 \\
\hline Choline Bitarrate & 2 & 0 \\
\hline FD\&C Bule Dye\#1 & 0.05 & 0 \\
\hline Total & 773.85 & 4057 \\
\hline
\end{tabular}


Table 2 List of oligonucleotide primer pairs used in RT-PCR analyses

\begin{tabular}{|c|c|}
\hline Name & Sequences \\
\hline H-GAPDH F & TGTGTCCGTCGTGGATCTGA \\
\hline H-GAPDH R & TTGCTGTTGAAGTCGCAGGAG \\
\hline M- $\beta$-actin F & AGCCATGTACGTAGCCATCC \\
\hline M- $\beta$-actin R & GCTGTGGTGGTGAAGCTGTA \\
\hline M-LncSHGL F & GACGCGTTTTCCTATCGTGC \\
\hline M-LncSHGL R & CCAGGCCCTCCATCATTTGT \\
\hline M-hnRNPA1 F & ATGTCTAAGTCCGAGTCTCCCA \\
\hline M-hnRNPA1 R & TGTTAGTGTTCCCCATTGCTC \\
\hline H-B4GALT1 F & AATCCATGTCTATAACTTCCT CAGT \\
\hline H-B4GALT1 R & AAGGATTCAGTTTATTCTCTGGCT \\
\hline H-hnRNPA1F & GGAACGCTCACGGACTGT \\
\hline H-hnRNPA1R & GTGGGCACCTGGTCTTTG \\
\hline
\end{tabular}

Table 3 List of the antibodies for western blot

\begin{tabular}{lllll}
\hline Antibody & Dilution & Cat.nr. & Source & Company \\
\hline P-AKT & $1: 2000$ & $\# 4060 \mathrm{~S}$ & Rabbit monoclonal IgG & CST \\
AKT & $1: 2000$ & $9272 \mathrm{~S}$ & Rabbit monoclonal IgG & CST \\
$\beta$-actin & $1: 1000$ & sc-47778 & Mouse monoclonal IgG & Santa Cruz \\
\hline
\end{tabular}

Figures 
A

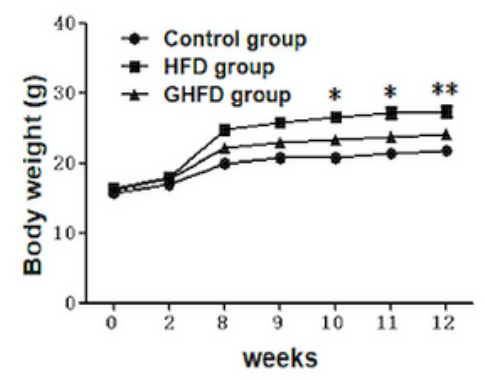

$\mathrm{D}$

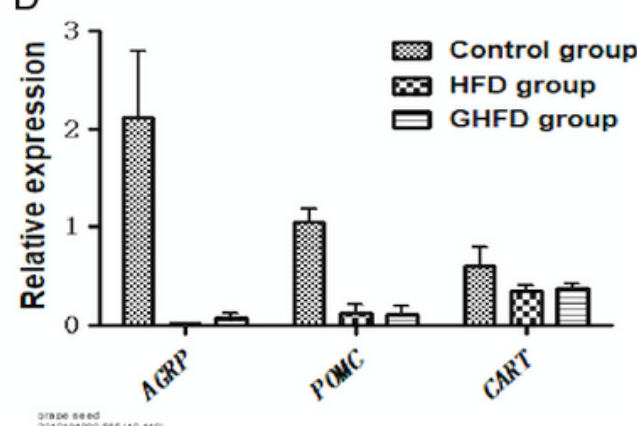

F

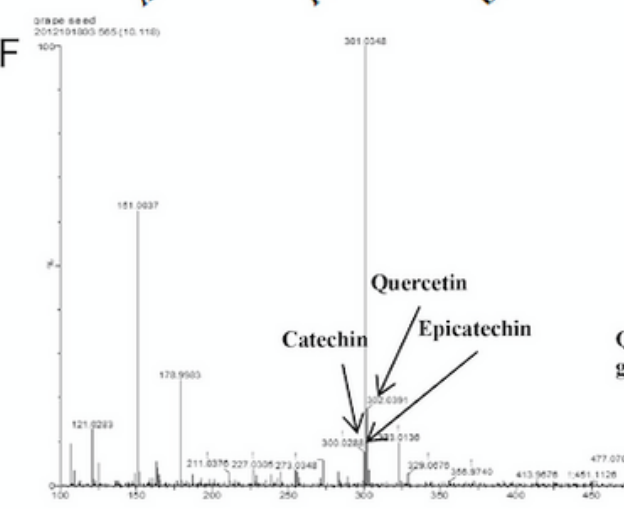

C

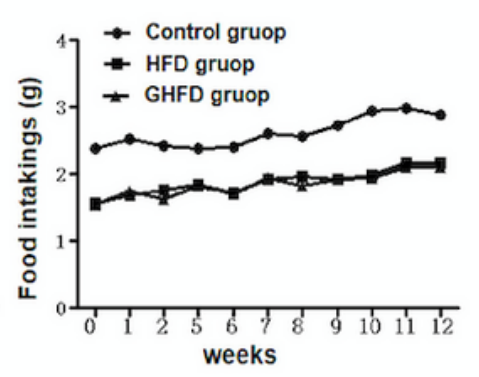

E
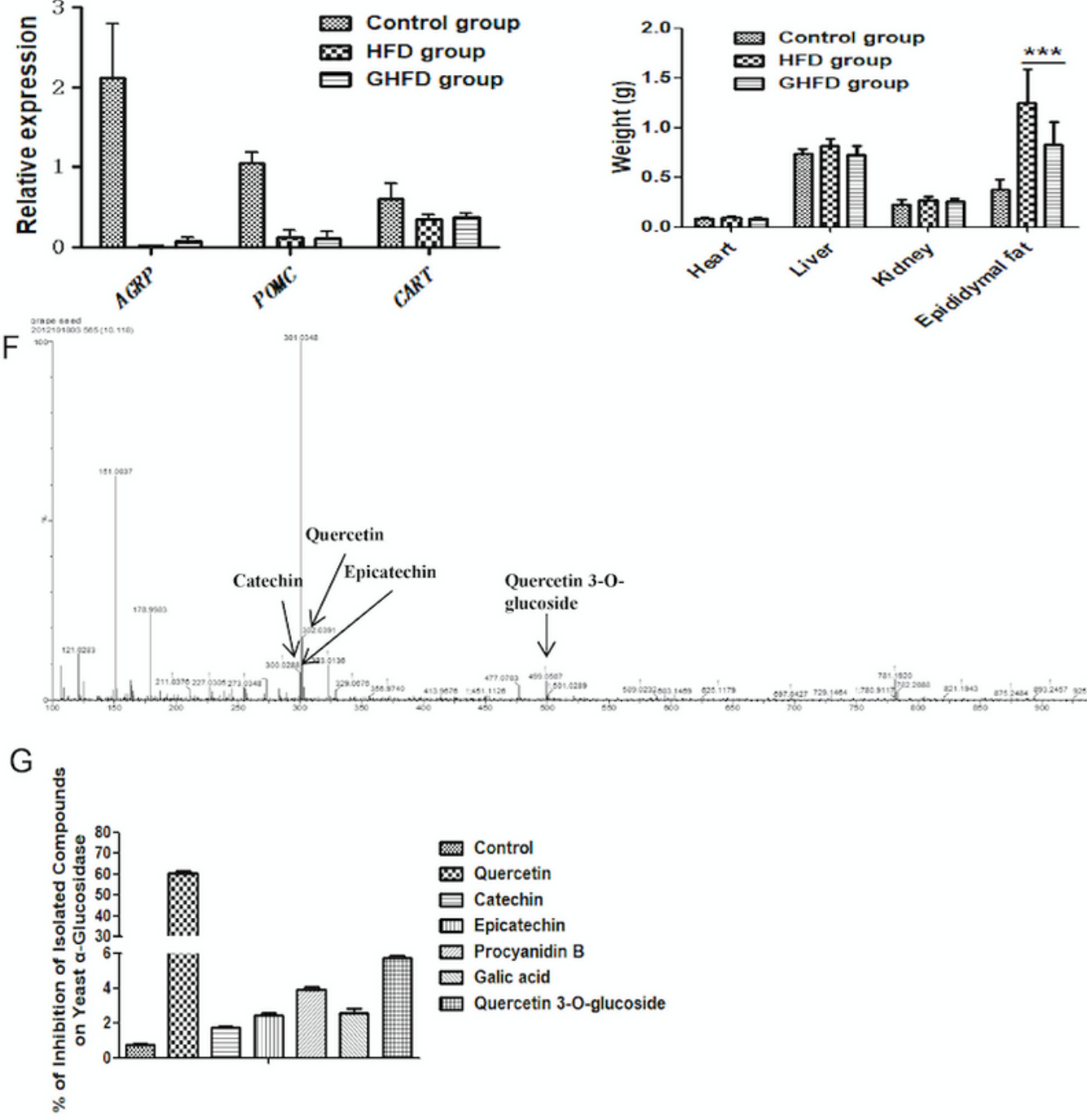

\section{Figure 1}

Grape pomace extract improves the symptoms of type 2 diabetes mellitus in mice fed a HFD. Grape pomace extract (GPE) improves the symptoms of type 2 diabetes mellitus in mice fed a HFD.(A) Body weight $(n=16)$ (GHFD group versus HFD group, **, $p<0.01 ; *, p<0.05)$, (B) fasting plasma glucose level $(n=16)$ (GHFD group versus HFD group, $* *, p<0.01 ; *, p<0.05)$, (C) food intake $(n=15),(D)$ expression 
of hypothalamic feed intake-related genes, (E) organ weight of each group of mice ( $n=16),(F)$ UPLC and Q-TOF-MS results and $(G)$ detect the efficiency of inhibition of a-galactosidase activity.

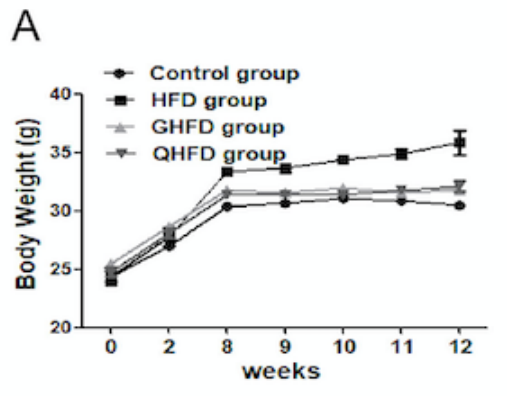

D

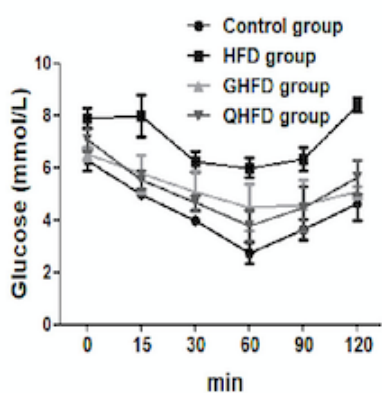

$\mathrm{H}$

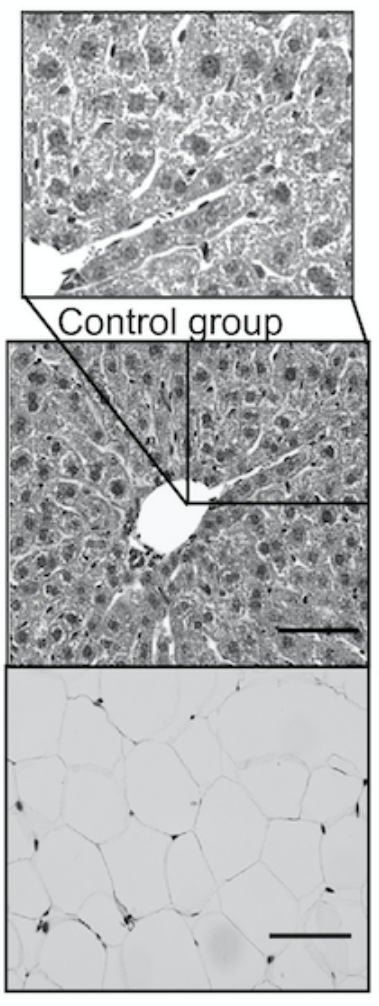

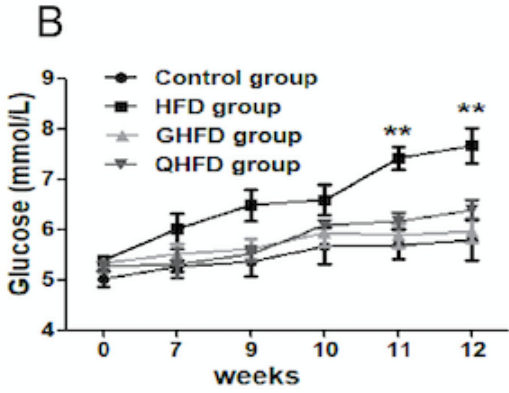

$\mathrm{F}$

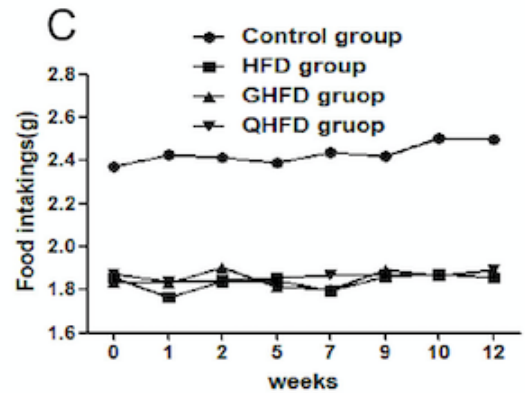

G
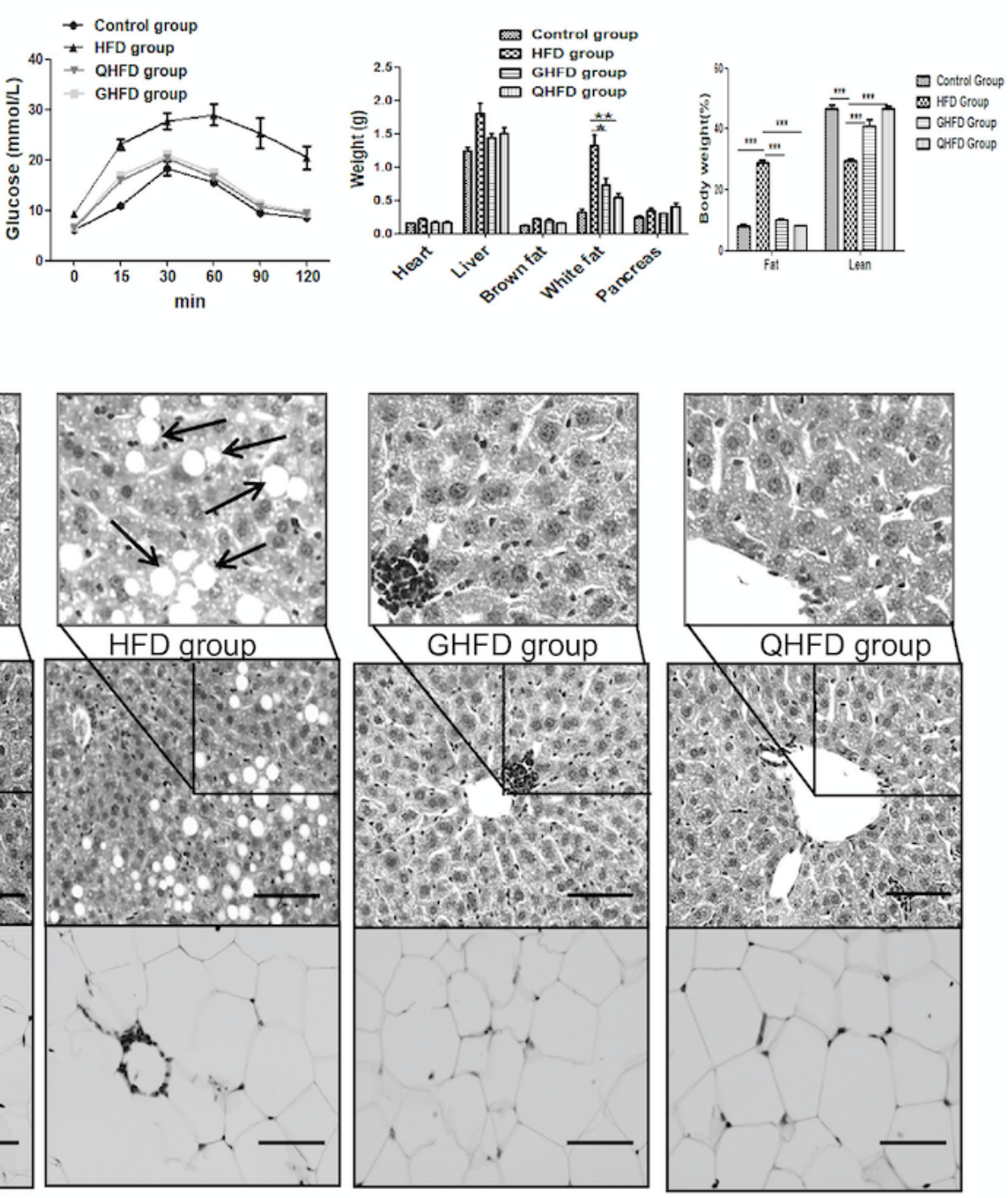

Figure 2

Quercetin can reduce HFD-induced type 2 diabetes mellitus in mice. Quercetin can reduce HFD-induced type 2 diabetes mellitus in mice. (A) Body weight $(n=16)$ (QHFD group versus HFD group, **, $p<0.01$; * $p$ $<0.05)$, (B) fasting plasma glucose level $(n=16)$ (QHFD group versus HFD group, $* *, p<0.01 ; *, p<0.05)$, 
(C) food intake ( $n=16)$, (D) ITT results of each group of mice $(n=16),(E)$ GTT results of each group of mice $(n=16)(F)$ organ weight of each group of mice $(n=16)$ (QHFD group versus HFD group, $* *, p<$ $0.01 ; *, p<0.05)$,, (G) body fat percentage and lean rate in mice and $(H)$ paraffin-embedded, hematoxylin and eosin stained sections of the liver and gonads $₫$ above the liver, and below the epididymal fat (magnification bar, 10 $\mu \mathrm{m}$ ).

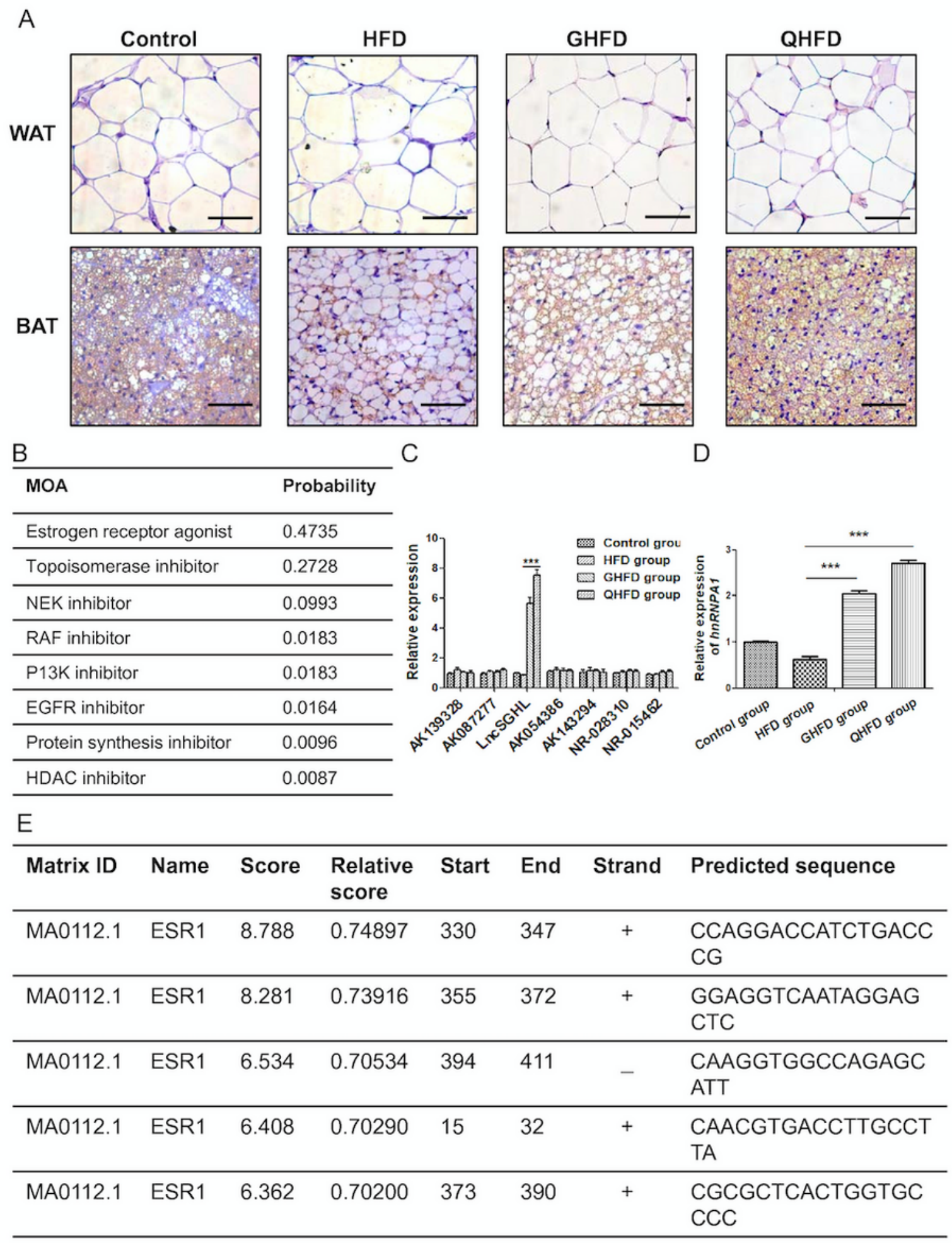

Figure 3 
Quercetin promotes IncSHGL expression via ER. Quercetin promotes IncSHGL expression via ER. (A) Immunohistochemical results that UCP1 of four groups WAT and BAT (magnification bar, $10 \mu \mathrm{m}),(\mathrm{B})$ quercetin MOA analysis (C) LncRNA expression in the liver, $(n=5)$ (QHFD group versus HFD group, $* *, p<$ $0.01 ; *, p<0.05)$, (D) hnRNPA1 expression in the liver, $(n=5)$ (GHFD group versus HFD group and QHFD group versus HFD group, $\left.* *, p<0.01 ;{ }^{*}, p<0.05\right)$, and $(E)$ prediction of the binding site between $E R$ and the IncRNA promoter region.

A

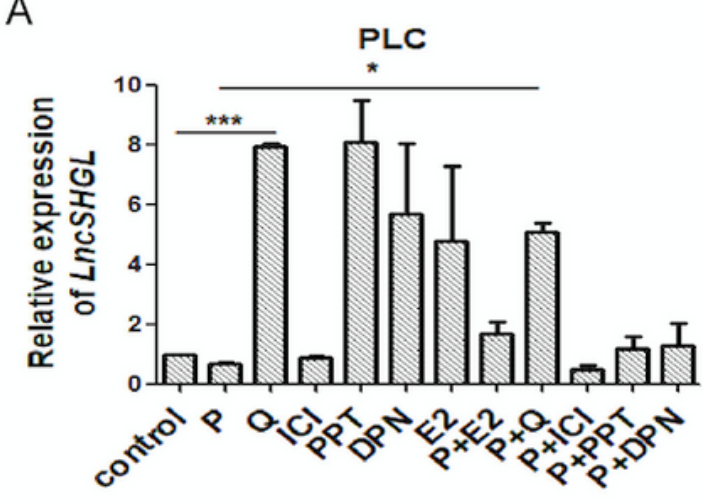

C

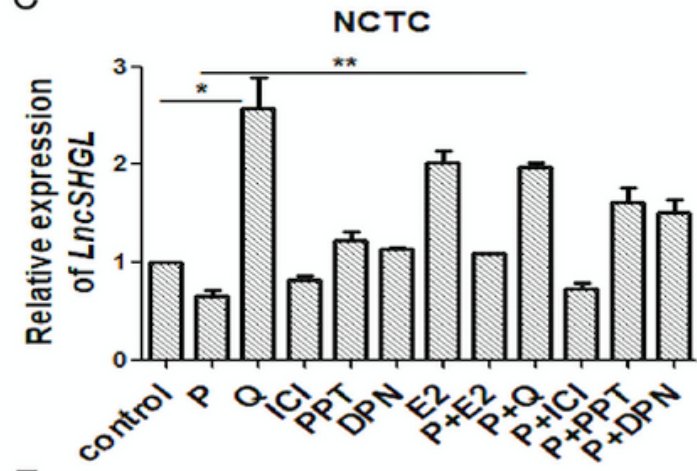

E

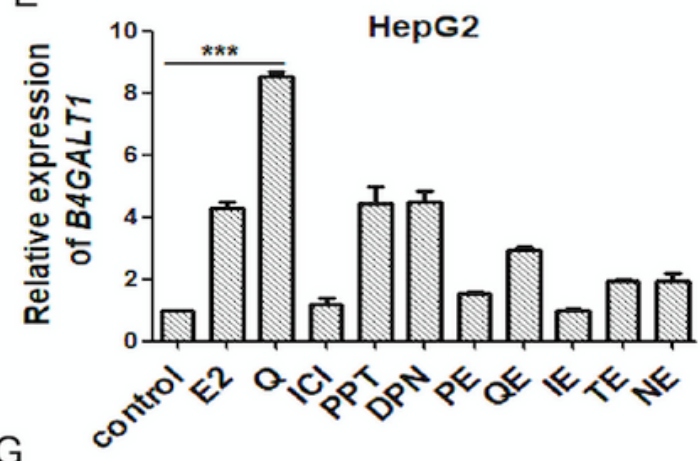

M Control PQ15min P15min PQ30min P30min M Q30min

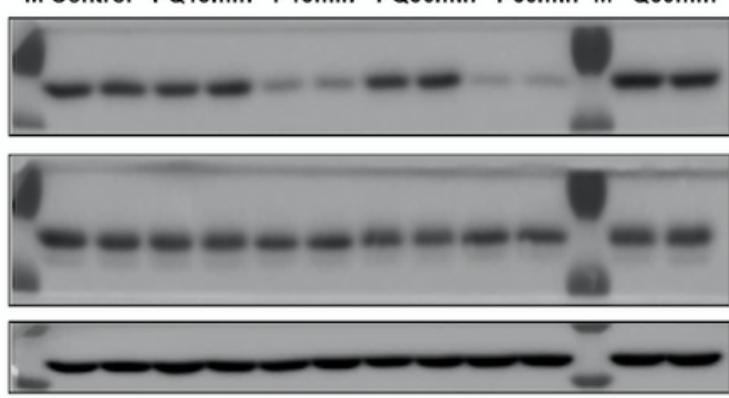

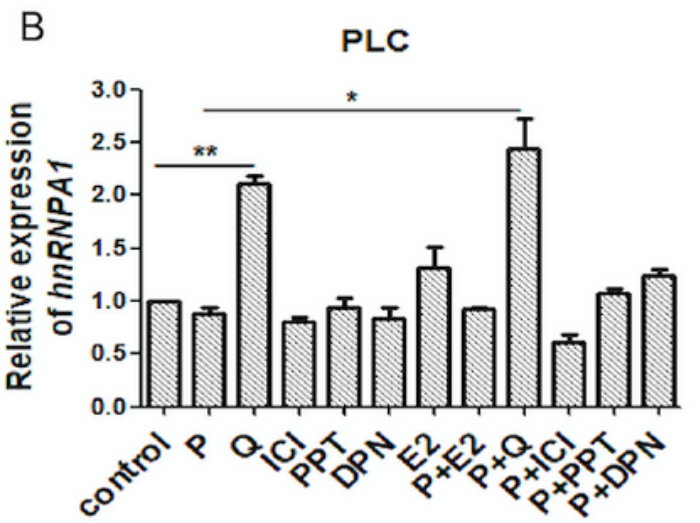

D

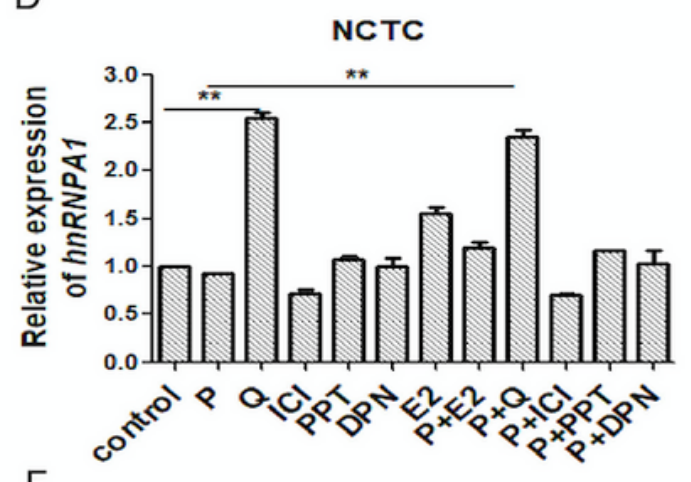

F
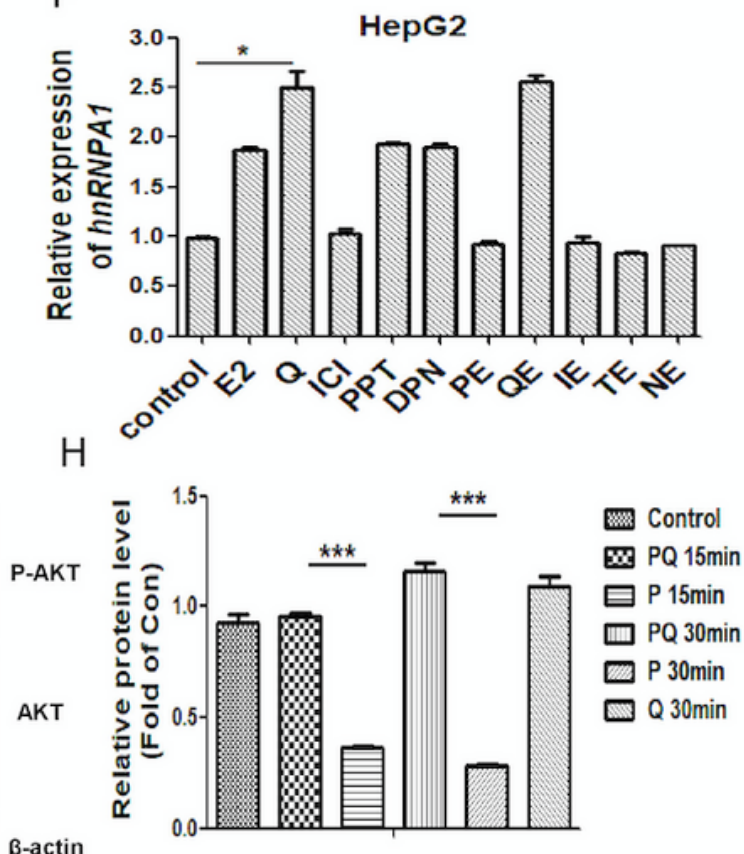

P-Akt/Akt 
Quercetin works with estrogen and ER agonists to increase IncSHGL expression. Quercetin works with estrogen and ER agonists to increase IncSHGL expression. (A) LncSHGL expression in primary hepatocytes after various treatments, $(n=5)$ (Control versus $Q$ and $P$ versus $P+Q, * *, p<0.01 ;{ }^{*}, p<0.05$ ), (B) hnRNPA1 expression in primary hepatocytes after various treatments, $(n=5)$ (Control versus $Q$ and $P$ versus $P+Q, * *, p<0.01 ; *, p<0.05)$, (C) IncSHGL expression in NCTC cells after various treatments, $(n=5)$ (Control versus $Q$ and $P$ versus $P+Q, * *, p<0.01 ; *, p<0.05)$, (D) hnRNPA1 expression in NCTC cells after various treatments, $(n=5)$ (Control versus $Q$ and $P$ versus $P+Q, * \star, p<0.01 ; *, p<0.05),(E)$ IncSHGL expression in HepG2 cells after various treatments, $(n=5)$ (Control versus $Q, * \star, p<0.01 ; *, p<0.05),(F)$ hnRNPA1 expression in HepG2 cells after various treatments, $(n=5)$ (Control versus $Q, * \star, p<0.01 ; *, p$ $<0.05)$, (G) changes in the phosphorylated AKT protein level and $(H)$ corresponding quantification of protein. 
A

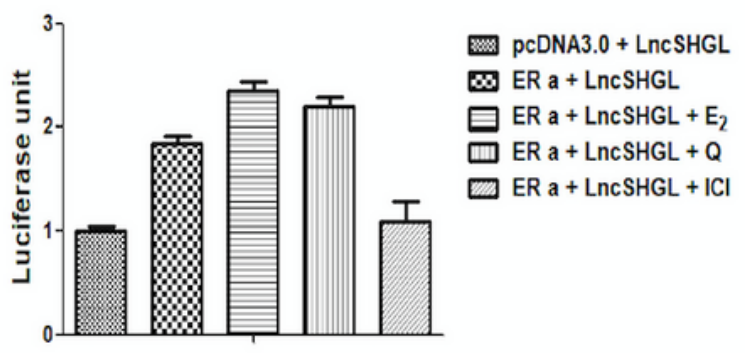

C

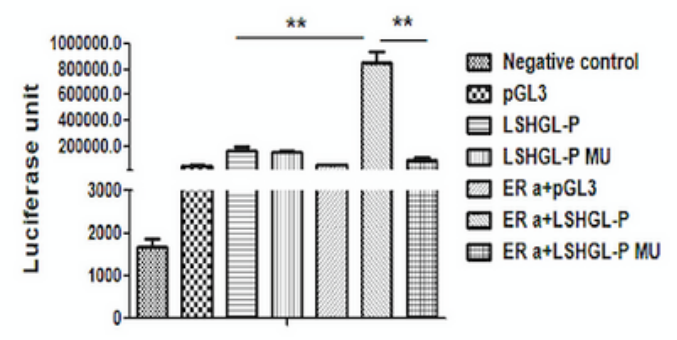

B

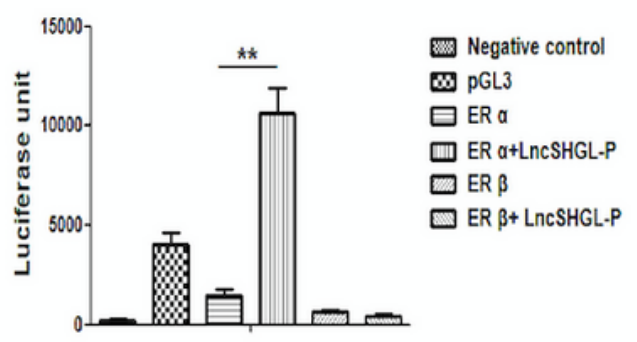

\section{D}

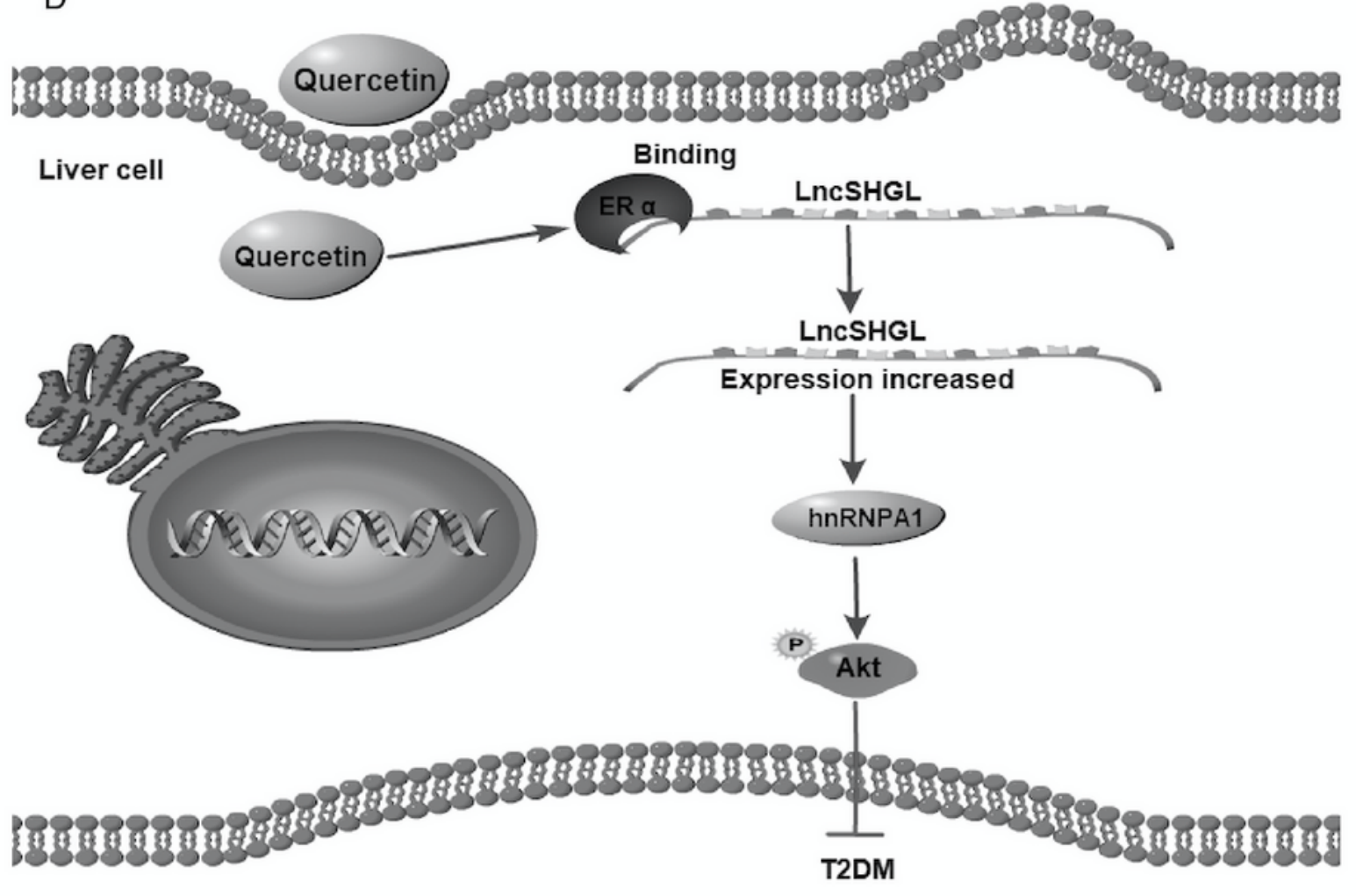

\section{Figure 5}

The LncSGHL promoter region binds to ERa. The LncSGHL promoter region binds to ERa. (A) Luciferase results, $(n=4)(E R a+L n c S H G L$ versus ER a + LncSHGL + E2 ; ER a + LncSHGL versus ER a + LncSHGL + $Q$ and ER $a+L n c S H G L$ versus ER $a+L n c S H G L+I C l, * \star, p<0.01 ; *, p<0.05)(B)$ Luciferase results, $(n=6)$ (ER a versus ER $a+$ LncSHGL-P, **, $p<0.01 ; *, p<0.05)$, (C) luciferase results, $(n=6)$ (LncSHGL-P versus ER $a+$ LncSHGL-P and ER $a+$ LncSHGL-P versus ER $a+$ LncSHGL-P MU, **, $p<0.01 ; *, p<0.05$ ) and (D) 
quercetin promoted ERa binding to the IncSHGL promoter region, thereby increasing IncSHGL expression and suppressing the symptoms of type 2 diabetes mellitus induced by a HFD.

\section{Supplementary Files}

This is a list of supplementary files associated with this preprint. Click to download.

- supplementary.docx 